\title{
Controversy in organophosphate poisoning management
}

This refers to the "Controversies in the management of organophosphate pesticide poisoning" by Bairy et al., published in IJ P of April 2007. ${ }^{[1]}$ The authors have stirred the controversy by raising the suspicion "whether one could use oximes at all in the management of organophosphate [OP] poisoning" by attempting to prop their proposition with inputs from other trials.

\section{We wish to submit that}

1. The authors have quoted that "patients with diethyl OP poisoning may particularly benefit from oxime therapy "[1] yet none of the trials quoted by the learned authors specify whether the poisoning was due to diethyl or dimethyl OP poison. Since Pralidoxime (PAM) is more effective in diethyl OP poisoning, its results will vary according to the subtype of poisoning.

2. In the randomized clinical trial (RCT) 1 , the sample size is too small $(n=21)$ for the evaluation effect of PAM in OP poisoning.

3. In RCT 2, the authors have admitted that the details of the type of study were not available and yet this has been considered for drawing the conclusion. In addition, the comparative groups in this trial were unequal.

4. The bible of pharmacology mentions "Atropine is virtually without effect against peripheral neuromuscular compromise which can be reversed by PAM." [2] The OP poison gets stored in fat and is released slowly later. This released OP poison can cause fresh aging of acetylcholinesterase (AchE). Hence PAM should be used continuously. "Oximes should be given until one can be assured of clearance of offending organophosphate". [2]

5. It seems that updated search has not been done by the authors for the clinical trials evaluating effectiveness of PAM in OP poisoning. Out of eight clinical trials referred by them, only two have been published after 2005.

On web search we found reports of two recently conducted trials:

In the first, 200 cases of OP poisoning were monitored. It was found that high dose regimen of PAM consisting of constant infusion of $1 \mathrm{gm} / \mathrm{h}$ for $48 \mathrm{~h}$ after $2 \mathrm{gm}$ loading dose, reduced the morbidity and mortality in moderately severe cases of acute OP poisoning ${ }^{[3]}$ confirming the importance of PAM in management of OP poisoning.

In the second study, where effectiveness of Oximes was studied in 58 patients of OP poisoning, the cases were classified under Nambas criteria into mild, moderate and severe and effectiveness of high dose of PAM ( $>4 \mathrm{gm}$ per day) was compared with low dose (<4gm per day). The authors concluded that the high dose PAM has valuable role in severe OP poisoning and along with good ventilatory support and aggressive atropinization, mortality could be definitely reduced. They recommend that high dose PAM should be administered without delay in patients with clinically moderate and severe OP poisoning, especially in centers where serum cholinesterase assay may not be available. ${ }^{[4]}$

Hence we have the reason to conclude that it is not justified to advice discontinuation of use of PAM in management of OP poisoning.

A.R. Kur undkar, S.R. J aiswal, V.R. Thawani Department of Pharmacology, Government Medical College, Nagpur - 440 003, India. E-mail: vijaythawani@rediffmail.com

\section{References}

1. Bairy KL, Vidyasagar S, Sharma S, Sammad V. Controversies in the management of organophosphate pesticide poisoning. Indian J Pharmacol 2007;39:714.

2. Taylor P, Anticholinesterase agents. In: Brunton LL, editor. Goodman and Gilman's The phramacological basis of therapeutics. $11^{\text {th }}$ ed. McGraw Hill Medical Publishing Division: New York; 2005. p. 210-1.

3. Pawar KS, Bhoite RR, Pillay CP, Chavan SC, Malshikare DS, Garad SG. Continuous pralidoxime infusion versus repeated bolus injection to treat organophosphorus pesticide poisoning: A randomized controlled trial. Lancet 2006;368:2136-41.

4. Eyer P, Szinicz L, Thiermann H, Worek F, Zilker T. Testing of antidotes for organophosphorus compounds: Experimental procedures and clinical reality. JAPI 2007;233:108-19. 